Becherglase mit 20 (bei 2 g. Salz), resp. 40 (bei $4 \mathrm{~g}$. Salz) C. C. destillirten $W$ asser anhaltend gemischt, dass das Gemenge eine Emulsions-Consistenz hat. Das Wasser darf nie $\ddot{i}$ ber $15^{\circ} \mathrm{C}$. haben, also mit eingesenktem Thermometer gemischt. Nach halbstïndiger Maceration wird klar filtrirt. In einem ganz trocknen, nicht zu weiten graduirten Cylinder giebt man 5 C.C. des Filtrats, lässt 7 C.C. Ammoniakflüssigkeit von $0,96 \mathrm{sp}$. G. oder $5 \mathrm{C}$. C. von $0,92 \mathrm{sp}$. G. vorsichtig zufliessen, so dass sich die Flüssigkeiten kaum mischen, und mischt dann durch ruhiges Neigen und Aufrichten. Die Flüssigkeit muss das etwa Ausgeschiedene vollständig wieder lösen oder klar bleiben. Fine schwache bleibende Opalescenz, die auf Zusatz eines weiteren 1/10 C. C. Ammoniakflüssigkeit verschwindet, berechtigt noch zu keiner ungünstigen Beurtheilung des Praeparats. Findet keine klare Lösung statt, so ist das Chinin. sulf. unrein. Ein starkes Schütteln beim Mischen der Flüssigkeiten darf wegen des hierdurch verursachten Verlustes an freiem Ammoniak nicht statt finden. Ueber die quantitative Beurtheilung der Verfälschung lese man in der Brochüre von Dr. Kerner nach. Wer $1 / 2$ Pfund oder darüber kauft, hat bei Zimmer in der Versiegelung des $\mathrm{Om}$ schlages mit dem Privat-Siegel des Hrn. Z. eine ziemliche Sicherheit, dennoch rathe ich, obige kleine Mühe nicht zu scheuen, um so mehr, als beim vorsichtigen Trocknen des. Rückstandes auf dem Filter kaum ein Verlust stattfindet.

\title{
Ueber das Einlassen von Glasstöpseln in Paraffin oder ähnliche Fettstoffe.
}

Von Gr. Glässner, Apotheker in Kassel.

Bekanntlich werden Glasstöpsel zu Gefässen, in denen Aetzalkalilaugen längere Zeit aufbewahrt werden sollen, häufig in Paraffin cingelassen, und erhält man anch aus chemischen 
Fabriken oft Gläser mit Lauge gefiullt (z. B. Normal-AetzkaliLauge), deren Stöpsel mit Paraffin oder wenigstens mit einem ähnlichen Fette eingelassen sind. Dieses quillt indess häufig, wenn es einigemale mit Aetzlauge in Berührung gekommen ist, zu einer schanmigen Masse auf, welche in die Lauge fliesst und darin eine geringe Trübung verursacht. Es ist nun von höchster Wichtigkeit, solche Lauge nicht etwa zu Fehling'scher Lösung zu verwenden, da sie dieses Reagens stark reducirt und so leicht zu Irrthümern Anlass geben kann. Auch bei Ausführung der Schiff'schen Probe ist es geboten, das Gemisch von weinsaurem Kupferoxyd und Kalilauge von zweifelhafter Reinheit stark aufkochen zu lassen, und es erst, wenn sich hierbei eine Trübung nicht zeigt, dem Harne zuzufügen.

\section{Ueber Tinet. Rhei aquosa.}

Von Dr. R. Mirus, Hofapotheker in Jona.

Im Novemberheft des vorigen Jahrgangs ist eine Vorschrift des Hofapotheker $F$ ischer in Dresden veröffentlicht, die - wie ich hierdurch ebenfalls bestätige, eine vortrefflich haltbare Tinct. Rhei aquosa liefert. Ich habe dieselbe im September vorigen Jahres bald nach meiner Rückkehr von Dresden dargestellt und finde sie heute noch ebenso unverändert.

Ich versuchte es nun, unter Hinweglassung des Borax und unter möglichst genauem Einhalten der Verhältnisse in der Vorschrift der Preuss. Pharmacopöe diese Tinctur darzustellen, verfuhr aber übrigens bezüglich der Bereitung genau nach den Angaben Fischer's und erhielt, wie ich erwartet hatte, eine ebenso haltbare Tinctur, wie die nach Fischer's Vorschrift bereitete es ist. *)

*) Ieh verweise hier zugieich auf die beiden Artikel ïber Tinct. Rhei aquosa von Dr. H. Ludwig in dem Januar - und von Dr. Enders im Septemberheft dieses Archivs, Jahrg. 1871; namentlich die von Enders 